\title{
Interface superconductivity found in single crystal
}

\section{Iron-based compound revives search for room-temperature superconductors.}

\section{BY EUGENIE SAMUEL REICH}

I $\mathrm{t}$ is one of the more counter-intuitive stunts in the repertoire of scientists who study superconductivity. Stack two materials, even insulators, on top of one another and voilà: a dramatic drop in electrical resistance can emerge between them. The negligible resistance of superconducting materials, sought for applications such as highly efficient electric grids, typically emerges at temperatures close to absolute zero - and only in certain materials. But physicists have shown that the stacking effect, called interface superconductivity, can occur at temperatures up to almost 80 kelvin.

Now, a researcher says that interface superconductivity can exist in a single crystal of an iron-based superconductor. The discovery will rejuvenate work on a family of compounds that first showed high-temperature superconductivity in 2008, but in recent years had seemed to disappoint.

"This is something conceptually very new," says Anvar Zakhidov, a physicist at the University of Texas at Dallas. "It raises fundamental questions that people will jump on."

The provocative result comes from a superconducting pioneer: Paul Chu of the University of Houston in Texas. In the 1980s, he helped to discover the first high-temperature superconductors, a family of copper-containing compounds (see 'All in the family'). In a paper posted on the arXiv preprint server on 30 August ${ }^{1}$, Chu and his colleagues now report their work on an iron-based crystal that generally superconducts at 30 kelvin - but that contains small regions that superconduct at 49 kelvin. Chu proposes that the effect is the result of many interfaces forming tens of nanometres apart within these regions (see 'At the interface').

If that is the case, it would be the first time

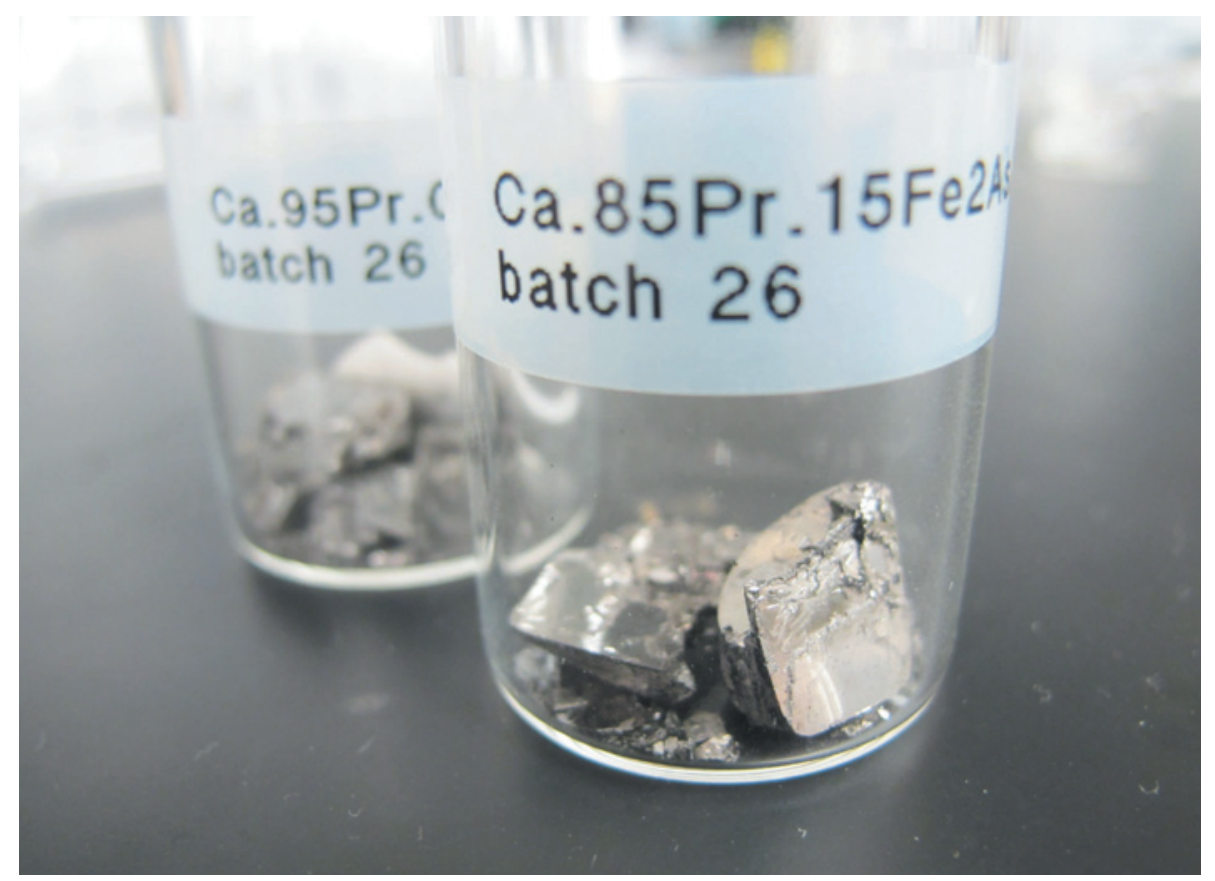

Crystals of an iron compound may contain regions that superconduct at higher temperatures than the bulk.

that interface superconductivity has been detected in a stable, stand-alone crystal, instead of at the interface between separate materials that often deteriorate on heating.

Physicists searching for a material that superconducts at room temperature (290 kelvin or more) have been disappointed before: almost three decades of work with the copper compounds have failed to produce one. After ironbased superconductors burst onto the scene in 2008, chemists began tweaking them, and quickly brought superconducting temperatures above 50 kelvin. But then progress plateaued.

That changed in February last year ${ }^{2}$, when physicists at Tsinghua University in Beijing reported interface superconductivity at about 80 kelvin - above the temperature of liquid nitrogen. They had layered iron selenide, the simplest iron-based superconductor, with strontium titanate, a well-known insulator. "This was stunning - the fact that we are able to construct new superconductors artificially," says Jean-Marc Triscone, a condensed-matter physicist at the University of Geneva in Switzerland.

But Chu's result goes further. He has reported the effect in a single crystal of a calcium-iron-arsenide compound doped with rare-earth elements, and suggests that the interfaces sit between regions that are enriched and depleted with arsenic.

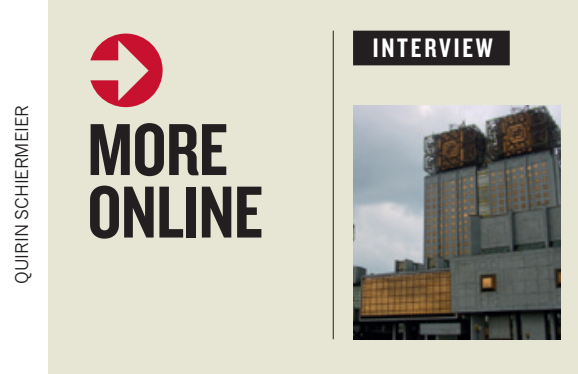

President of Russian Academy of Sciences hits out at political meddling in research go.nature. com/l6w6re

\section{MORE NEWS}

- Relic radiation suggests the Universe may not be flat go.nature.com/szagdh - Earth will leave the Sun's 'habitable zone' in 1.75 billion years go.nature.com/ rqpnon

- Rover sees no sign of methane in Martian atmosphere go.nature.com/jdrgxk

\section{NATURE PODCAST}

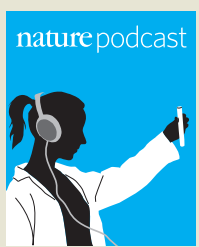

A carbon-nanotube computer, saving species by tweaking their genes, and early Earth's similarity to lo nature. com/nature/podcast 
If correct, the result would show that interface superconductivity is consistent with a mechanism first advanced in 1973, which helps to explain low-temperature superconductivity. Proposed by US physicist John Bardeen and his colleagues, it posits that superconducting electron pairs are held together by an exciton, a particle produced by the scattering of electrons off a boundary. This does not explain all hightemperature superconductivity, but exploring it further might shed light on mechanisms that do.

Superconductivity is a field with a long history of mistaken claims and false hopes, and so it is unsurprising that Chu's result is provoking controversy. Critics say that it is difficult to measure superconductivity along narrow interfaces, let alone inside a crystal. And Johnpierre Paglione, a condensed-matter physicist at the University of Maryland in College Park, is dubious for a different reason. In 2012, he and his team reported ${ }^{3}$ a higher-than-expected superconducting temperature for regions within the same material. But in a paper posted on arXiv on 13 September ${ }^{4}$, the researchers say that what they saw is a bulk property of these regions,

\section{ALL IN THE FAMILY}

Superconducting temperatures have risen as new classes of material have been found.

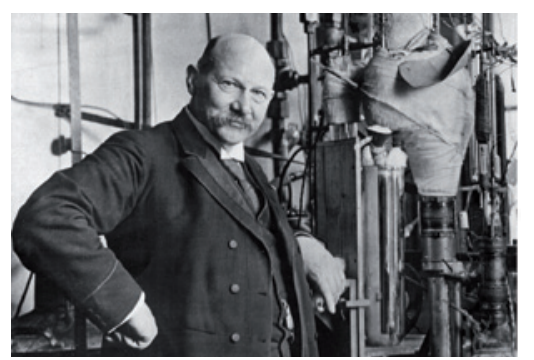

19114.2 kelvin: Mercury. Superconductivity first discovered by Heike Kamerlingh Onnes (pictured).

198630 kelvin: Barium lanthanum copper oxide. First copper-based hightemperature superconductor.

1993133 kelvin: Mercury barium calcium copper oxide. Record hightemperature superconductor.

\section{6 kelvin: Lanthanum} iron arsenide. First iron-based hightemperature superconductor.

200850 kelvin: Layered insulating and metallic copper oxide. One of the first interface-enhanced superconductors.

201349 kelvin: Calcium iron arsenide doped with rare-earth elements. Claimed single-crystal interface enhanced superconductor.

\section{AT THE INTERFACE}

Nanoscale layering in an iron-based crystal could enhance its superconductivity.

Bulk of crystal superconducts at
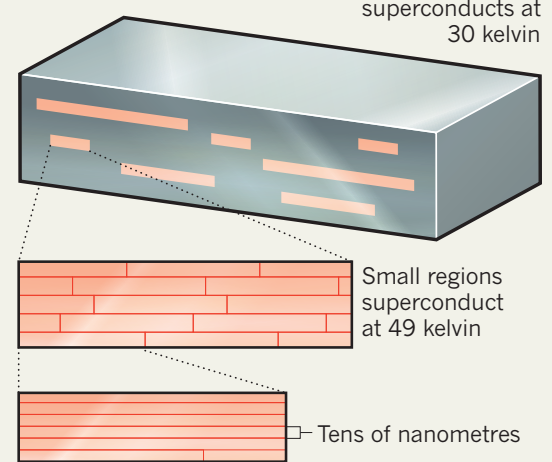

Nanoscale layers may be responsible for the enhancement

caused by the doping with rare-earth elements, rather than an interface effect.

If the superconductivity was related to interfaces, Paglione says, he would not expect it to disappear when the doping is reduced - which it does. Instead, Paglione suggests that strain in certain regions of the crystal enhances superconductivity. Chu responds that doping above a certain level helps to create interfaces by pushing arsenic atoms out of the structure.

Ivan Bozovic, a physicist at Brookhaven National Laboratory in Upton, New York, who in 2008 created one of the first interfaced superconductors, raises another possibility. $\mathrm{He}$ says that the superconductivity might not be an interface effect; instead, it could be attributable to a structural change inherent to the arsenicdepleted regions. He would like to see whether Chu can isolate these regions to study them further - and perhaps use them to create other interfaces. "We've been looking for new superconductors for 100 years and interface superconductivity is a new trick," says Bozovic, "so there's a lot of room for new combinations."

1. Wei, F. Y. et al. Preprint at http://arxiv.org/ abs/1309.0034 (2013)

2. Wang, Q.-Y. et al. Chin. Phys. Lett. 29, 037402 (2012).

3. Saha, S. R. et al. Phys. Rev. B 85, 024525 (2012).

4. Saha, S. R. et al. Preprint at http://arxiv.org/ abs/1309.3576 (2013).

\section{CORRECTIONS}

The scale on the graph showing German science spending in the News story 'Germany hits science high' (Nature 501, 289-290; 2013) had an incorrect scale. It should have run from $€ 0$ to $€ 15$ billion not $€ 0-1.5$ billion. And the News story 'Grass gets greener' (Nature 501, 291; 2013) incorrectly identified the grain that produced high yields when grown in a field that previously hosted Brachiaria grasses. It was maize (corn), not wheat. 